\title{
NÂNG CAO Độ CHÍNH XÁC CỦA MÔ HÌNH SỐ ĐỊA HİNH BĂNG PHƯƠNG PHÁP LỌC KALMAN VÀ PHÉP LÀM TROON RAUCH-TUNG-STRIEBEL
}

\author{
DƯO'NG THÀNH TRUNG ${ }^{(1)}$, ĐÀO VĂN KHÁNH(1), \\ TRƯO'NG MINH HÙNG ${ }^{(1)}$, NGUYẼ̃N ĐỨC MANHH ${ }^{(2)}$ \\ (1)Trường Đại học Mỏ - Địa chất Hà Nội \\ ${ }^{(2)}$ Viện Khoa học Đo đạc và Bản đồ
}

\section{Tóm tắt:}

Mô hình số độ cao (DEM) là dũ liệu cần thiết cho các ứng dụng về nghiên cứu bề mặt Trái đất, tài nguyên thiên nhiên và môi trường. Trên thực tế, có hai cách chính để thành lập DEM là: (1) Phương pháp đo đạc truyền thống trong đó sử dụng các loại máy móc trắc địa để đo đạc trực tiếp bề mặt Trái đất, và (2) Phương pháp viễn thám, trong đó việc thu nhận dữ liệu DEM được thực hiện bằng các thiết bị đặt trên các thiết bị bay hoặc vệ tinh. Thực tế thấy rằng phương pháp đo đạc truyền thống thường cho ra sản phẩm DEM có độ chính xác cao (từ $2 \mathrm{~cm}-1 \mathrm{~m}$ ) nhưng chi phí sản xuất cao và năng suất thấp. Ngược lại, phương pháp viễn thám, đặc biệt là sử dụng các dữ liệu vệ tinh có giá thành thấp, năng suất cao, nhưng sản phẩm có độ chính xác thấp (từ $5 m-30 m$ ). Từ vấn đề trên, chúng tôi đề xuất hướng nghiên cứu nhằm nâng cao hiệu quả thành lập DEM bằng phương pháp tích hợp dữ liệu DEM vệ tinh và mặt đất thông qua phép lọc Kalman và phép làm trơn Rauch-TungStriebel.

\section{Mở đầu}

Bằng công nghệ viễn thám, các mô hình số độ cao (DEM) có thể được thành lập trên phạm vi rộng lớn, thậm chí toàn cầu để phục vụ cho các mục đích nghiên cứu Trái đất, môi trường và quản lý tài nguyên thiên nhiên. Các DEM trên phạm vi toàn cầu có thể được kể đến như SRTM, ASTER GDEM, ALOS-DEM. Với các dư liệu DEM hiện có như trên, người dùng có thể khai thác sử dụng gần như ở bất kỳ nơi đâu với chi phí rẻ, thậm chí miễn phí. Tuy vậy độ chính xác của các DEM này vẫn chưa thể đáp ứng được nhu cầu sử dụng cho nhiều ứng dụng. Các nghiên cứu chỉ ra rằng độ chính xác độ cao của SRTM DEM và ASTER GDEM vào khoảng từ 10-15m [1]. Độ chính xác của ALOS DEM khoảng từ 3$5 \mathrm{~m}$ [2]. Để đáp ứng yêu cầu sử dụng, việc nâng cao độ chính xác của các DEM là cần thiết. Một trong những phương pháp nhằm nâng cao độ chính xác của DEM là sử dụng các phương pháp xử lý số liệu hoặc bằng việc kết hợp $D E M$ được xây dựng bằng công nghệ viễn thám với các số liệu đo đạc mặt đất. Nicholas J.T và nnk [3] giới thiệu phép làm trơn/lọc $\mathrm{DEM}$ thành lập bằng công nghệ Lidar thông qua thuật toán hồi quy và wavelets rời rạc. Kết quả nghiên cứu cho rằng phương pháp đề xuất có thể lọc nhiễu và nâng cao độ chính xác của DEM. Ping Wang, (1998) [4] đề xuất phương pháp lọc Kalman hai chiều để nâng cao độ chính xác của $D E M$. Kết quả nghiên cứu chỉ ra rằng phương pháp đề xuất có thể giảm được 60$70 \%$ nhiễu so với mô hình nguyên thủy. Tuy vậy hạn chế của nghiên cứu là các thí nghiệm chỉ được thực hiện trên DEM mô phỏng vì vậy mô hình toán học của thuật toán cần cải tiến để phù hợp với đa dạng các loại $D E M$ và các điểm đo mặt đất ngẫu nhiên. 
Trong nghiên cứu này, để nâng cao độ chính xác của một DEM đã có, trước hết các điểm đo với độ chính xác cao hơn được thu thập. Thông qua phép lọc Kalman và phép làm trơn Rauch-Tung-Striebel DEM nguyên thủy và các điểm đo mới được kết hợp nhằm cải thiện độ chính xác của $D E M$ nguyên thủy.

\section{Lý thuyết phép lọc Kalman và phép làm trơn Rauch-Tung-Striebel}

Phép lọc Kalman (KF) [5] được sử dụng rộng rãi trong việc xử lý số tín hiệu số và dữ liệu đa cảm biến. Mục đích của KF là xác định ước lượng tin cậy nhất của các đại lượng cần xác định dựa trên nguyên lý tối thiểu hóa ma trận hiệp phương sai tương ứng. Để áp dụng lý thuyết $\mathrm{KF}$, các mô hình toán học bao gồm mô hình trạng thái và mô hình trị đo phải được xây dựng ở dạng như sau:

Mô hình hệ thống:

$$
x_{k}=F_{k-1 ; k} x_{k-1}+B_{k} u_{k}+w_{k}
$$

Mô hình trị đo:

$$
z_{k}=D_{k} x_{k}+v_{k}
$$

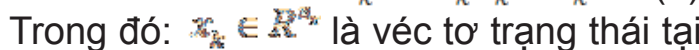
$k, F_{k-1: k}: R^{n_{x}} \times R^{n_{v}} \rightarrow R^{n_{x}}$ là ma trận hệ số chuyển đổi trạng thái từ $k-1$ đến $k$, 将

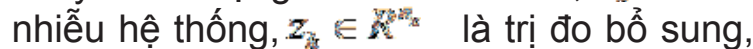
$D_{k}: R^{n_{x}} \times R^{n_{v}} \rightarrow R^{n_{z}}$ là ma trận liên hệ giữa trị đo bổ sung và véc tơ trạng thái, và 慗 $\in \mathcal{K}^{2}$ là nhiễu của trị đo bổ sung.

KF là một phép ước lượng lặp, chu trình tính toán có thể chia thành hai bước chính:

Bước 1: Dự đoán hay cập nhật trạng thái: Dựa vào mô hình toán học hệ thống trong công thức (1), Véc tơ trạng thái và ma trận hiệp phương sai tương ứng ở thời điểm $k$ được ước tính dựa trên các tham số đó tại $k-1$.

$$
\begin{aligned}
& x^{-}{ }_{k}=F_{k-1 ; k} x_{k-1}+B_{k} u_{k} \\
& P_{k}^{-}=F_{k-1 ; k} P_{k-1} F_{k-1 ; k}^{T}+Q_{k}
\end{aligned}
$$

Bước 2: Cập nhật trị đo: Khi có các trị đo bổ sung, vector trạng thái và ma trận hiệp phương sai được cập nhật dựa theo công thức sau:

$$
\begin{aligned}
& K_{k}=P_{k}^{-} D_{k}^{T}\left[D_{k} P_{k}^{-} D_{k}^{T}+R_{k}\right]^{-1} \\
& \hat{x}_{k}=\hat{x}_{k}^{-}+K_{k}\left(z_{k}-D \hat{x}_{k}^{-}\right) \\
& P_{k}=P_{k}^{-}-K_{k} D_{k} P_{k}^{-}
\end{aligned}
$$

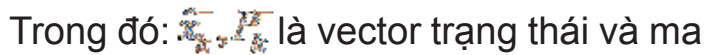
trận hiệp phương dự đoán sai tại thời điểm $k, \quad \hat{x}_{k-1}, P_{k-1}$ là vector trạng thái và ma trận

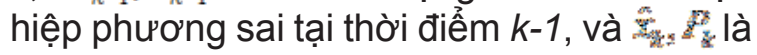
vector trạng thái và ma trận hiệp phương sai cập nhật tại thời điểm $k ; Q_{k}=\left\{\mathrm{w}_{k} \mathrm{w}_{k}^{T}\right\}$.

Mặc dù đã được ứng dụng rộng rãi trong nhiều lĩnh vực, $K F$ vẫn có hạn chế là chỉ cho ước lượng tốt nhất khi có các trị đo bổ sung. Trong trường hợp không có trị đo bổ sung, ước lượng của $\mathrm{KF}$ hoàn toàn dựa vào mô hình hệ thống, điều này làm giảm tính tin cậy của phép ước lượng. Để khắc phục tình trạng này phép làm trơn Rauch-TungStriebel [6] đã được đề xuất với nội dung như sau.

Phép ước lượng trơn RTS sử dụng ước lượng thuân và đảo để tính ước lượng tối ưu. Ước lượng thuận chính là phép lọc KF và ước lượng đảo được dựa trên nguyên lý xác xuất cực đại. Vector trạng thái và ma trận hiệp phương sai trong phép ước lượng trơn RTS được xác định theo các công thức sau:

$$
\begin{aligned}
& \hat{x}_{k \mid N}=\hat{x}_{k}+C_{k}\left[\hat{x}_{k+1 \mid N}-\hat{x}_{k+1}\right] \\
& P_{k \mid N}=P_{k}+C_{k}\left[P_{k+1 \mid N}-P_{k+1}\right] C_{k}^{T}
\end{aligned}
$$

Trong đó $\hat{x}_{k \mid N}$ và $P_{k \mid N}$ là vector trạng thái và ma trận hiệp phương sai tại thời điểm $k$ với các thông tin cung cấp đến $N(k \leq N)$; 管 và $P_{\text {: }}$ là vector trạng thái và ma trận hiệp phương sai cung cấp bởi EKF tại thời điểm $k ; C_{k}$ là ma trận tương quan, được xác định theo công thức:

$$
C_{k}=P_{k} F_{k ; k+1}^{T} P_{k+1}^{-1}
$$

3. Xây dựng mô hình toán học cho 


\section{phép lọc mô hình số độ cao}

Để nâng cao tính hiệu quả của phép lọc, trước hết, cấu trúc DEM được sắp xếp lại với số thứ tự các mắt lưới được đánh tuần tự theo cấu trúc zigzac (xem hình 1).

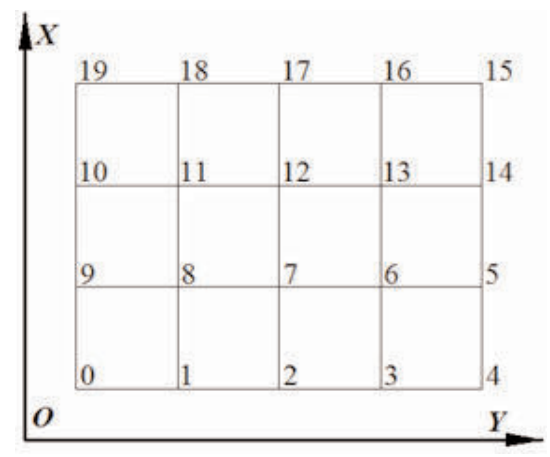

Hinh 1

\subsection{Mô hình hệ thống}

Xét trong một ô lưới, quan hệ độ cao giữa các mắt lưới có thể được thiết lập như sau: (xem hinh 2)

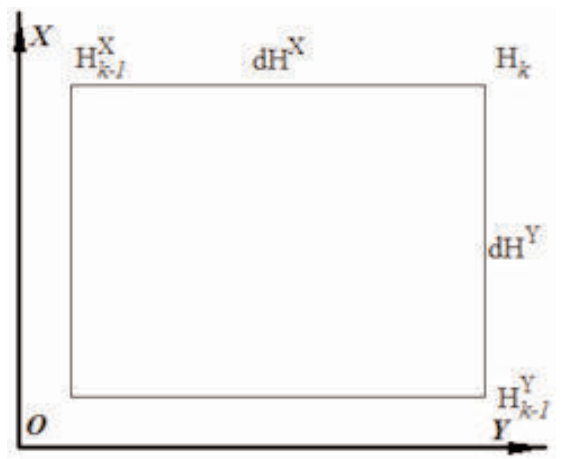

Hinh 2

$$
\begin{aligned}
H_{k}^{X} & =H_{k-1}^{X}+d H^{X}+w^{X} \\
H_{k}^{Y} & =H_{k-1}^{Y}+d H^{Y}+w^{Y} \\
H_{k} & =a H_{k}^{X}+b H_{k}{ }^{Y}
\end{aligned}
$$

Trong đó: $H_{k}$ là độ cao tại điểm đang xét

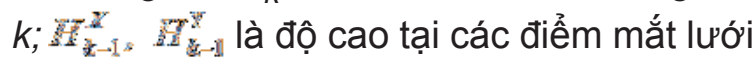
trước đó theo chiều $X$ và $Y ; d H^{x}, d H^{y}, w^{x}, w^{y}$ là chênh cao và nhiễu theo các chiều $X, Y$ tương ứng; $a, b(a+b=1)$ là trọng số độ cao theo hướng $X$ và $Y$

Từ công thức (11) và (12) chúng ta có thể viết dưới dạng ma trận như sau:

$$
\left(\begin{array}{l}
H_{k}^{X} \\
H_{k}^{Y}
\end{array}\right)=\left(\begin{array}{ll}
1 & 0 \\
0 & 1
\end{array}\right)\left(\begin{array}{l}
H_{k-1}^{X} \\
H_{k-1}^{Y}
\end{array}\right)+\left(\begin{array}{l}
d H^{X} \\
d H^{Y}
\end{array}\right)+\left(\begin{array}{c}
w^{X} \\
w^{Y}
\end{array}\right)
$$

Công thức (14) có thể được viết lại ở dạng của công thức (1) với:

$$
x_{k}=\left(\begin{array}{l}
H_{k}^{X} \\
H_{k}^{Y}
\end{array}\right) ; F_{k-1 ; k}=\left(\begin{array}{ll}
1 & 0 \\
0 & 1
\end{array}\right) ; B_{k}=\left(\begin{array}{l}
d H^{X} \\
d H^{Y}
\end{array}\right) ; \mathrm{w}_{k}=\left(\begin{array}{l}
w^{X} \\
w^{Y}
\end{array}\right) ; u_{k}=1
$$

\subsection{Mô hình trị đo}

Giả sử có các điểm đo ngẫu nhiên trong khu đo (P1, P2, P3) nhu minh họa trong hình 3 , các mắt lưới của các ô lưới có chứa các điểm đo được cập nhật độ cao sơ bộ theo nguyên tắc trung bình trọng số (xem hình3):

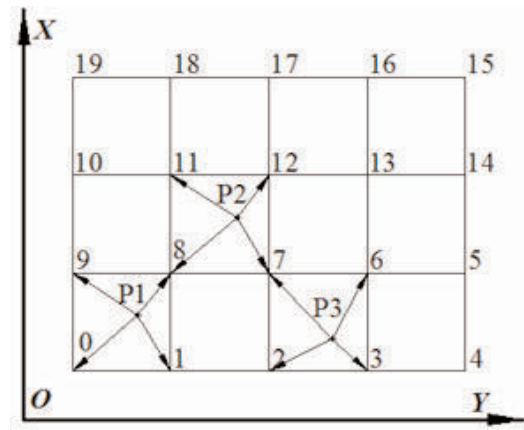

Hinh 3

$$
\begin{gathered}
H_{k}^{\sim}=\frac{P_{k} H_{K}+P_{P} H_{P}}{P_{k}+P_{P}} \\
P_{k}^{\sim}=\left(\frac{1}{P_{k}}+\frac{1}{P_{P}}\right)^{-1}
\end{gathered}
$$

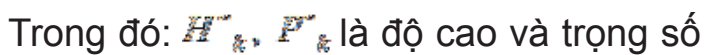
tại điểm $k$ được cập nhật sơ bộ dựa vào điểm đo $P ; P_{p}$ là trọng số về độ cao của điểm đo $P$, được tính dựa vào khoảng cách giữa điểm $P$ và $k$.

Sau khi được cập nhật sơ bộ, mô hình trị đo tại các điểm mắt lưới được xây dựng như sau:

$$
H_{k}^{\sim}=\left(\begin{array}{ll}
a & b
\end{array}\right)\left(\begin{array}{l}
H_{k}^{X} \\
H_{k}^{Y}
\end{array}\right)+v_{k}
$$

Công thức (19) có thể được viết dưới dạng của công thức (2) với:

$$
z_{k}=H_{k}^{\sim} ; D=(a b) ; E\left\{v_{k} v_{k}^{T}\right\}=P_{k}^{\sim}
$$


Như vậy sau khi xây dựng được mô hình toán học của mô hình số độ cao cho phép lọc Kalman, các bước tính toán dựa trên thuật toán của phép lọc Kalman và phép làm trơn RTS được thực hiện tuần tự các bước theo các công thức từ (3) đến (9).

\section{Tính toán thực nghiệm}

Các số liệu cho tính toán thực nghiệm là khu đo có diên tích 16ha tại Thành phố Lạng Sơn, tỉnh Lạng Sơn. Bản đồ vị trí khu thực nghiệm được thể hiện ở hình 4 (xem hình 4). Trên khu thực nghiệm, một mô hình số được xây dựng dựa trên các điểm đo địa hình theo tiêu chuẩn của bản đồ địa hình tỷ lệ 1:1000 khoảng cao đều $1 \mathrm{~m}$, với khoảng cách điểm mia 20-30m được dùng làm DEM chuẩn, sai số độ cao của DEM chuẩn (DEM0) theo tiêu chuẩn là $10 \mathrm{~cm}$ (xem hình 5). Một mô hình trích xuất từ ASTER GDEM tại khu thực nghiệm với kích thước mắt lưới $30 \mathrm{~m} \times 30 \mathrm{~m}$ được sử dụng làm DEM gốc (DEM1) với nhiễu độ cao được cho là $1 \mathrm{~m}$ (xem hình 6). Để tại một DEM thử nghiệm, các điểm thử nghiệm được trích ra từ điểm đo của bản đồ chuẩn có mật độ điểm giảm đi 10 lần (xem hình 7), ký hiệu là DEM2. Mô hình DEM thử nghiệm sau đó được kết hợp với các điểm đo trong mô hình DEM gốc thông qua phép lọc Kalman và phép làm trơn RTS để được DEM sau xử lý (DEM3) (xem hình 8). Việc tính toán được thực hiện trên phần mềm Pointer, do các tác giả viết bằng ngôn ngữ lập trình Visual $\mathrm{C \#}$. DEM sau xử lý được so sánh với mô hình chuẩn để phân tích, đánh giá tính hiệu quả. Để đánh giá tính hiệu quả của phương pháp đề xuất, các mô hình DEM được nội suy lại với cùng kích thước và số lượng các mắt lưới là 100x100. Sai số trung phương độ cao của mô hình sau đó được tính dựa trên độ cao của tất cả các mắt lưới so với mô hình chuẩn theo công thức sau:

$$
m_{D E M j}=\sqrt{\frac{\sum_{i=1}^{n} \Delta h_{i j}^{2}}{n}}
$$

Trong đó:

$\Delta h_{j i}=D E M_{j i}-D E M_{0 i}(j=1 \rightarrow 3 ; i=1 \rightarrow n)$

$n$ là số mắt lưới trong mô hình nội suy.

Kết quả phân tích độ chính xác của các DEM được trình bày ở bảng (xem bảng1).

Từ kết quả thực nghiệm chúng ta thấy rằng nếu coi sai số của $\mathrm{DEM}$ chuẩn là không đáng kể, độ chính xác của ASTER GDEM (DEM1) vào khoảng $8.6 \mathrm{~m}$. Sau khi giảm mật đo điểm đo của DEM chuẩn đi 10 lần, độ chính xác của DEM thử nghiệm (DEM2) là $6.1 \mathrm{~m}$. Với sự kết hợp của DEM1 và DEM2 dựa trên phương pháp đã đề xuất,

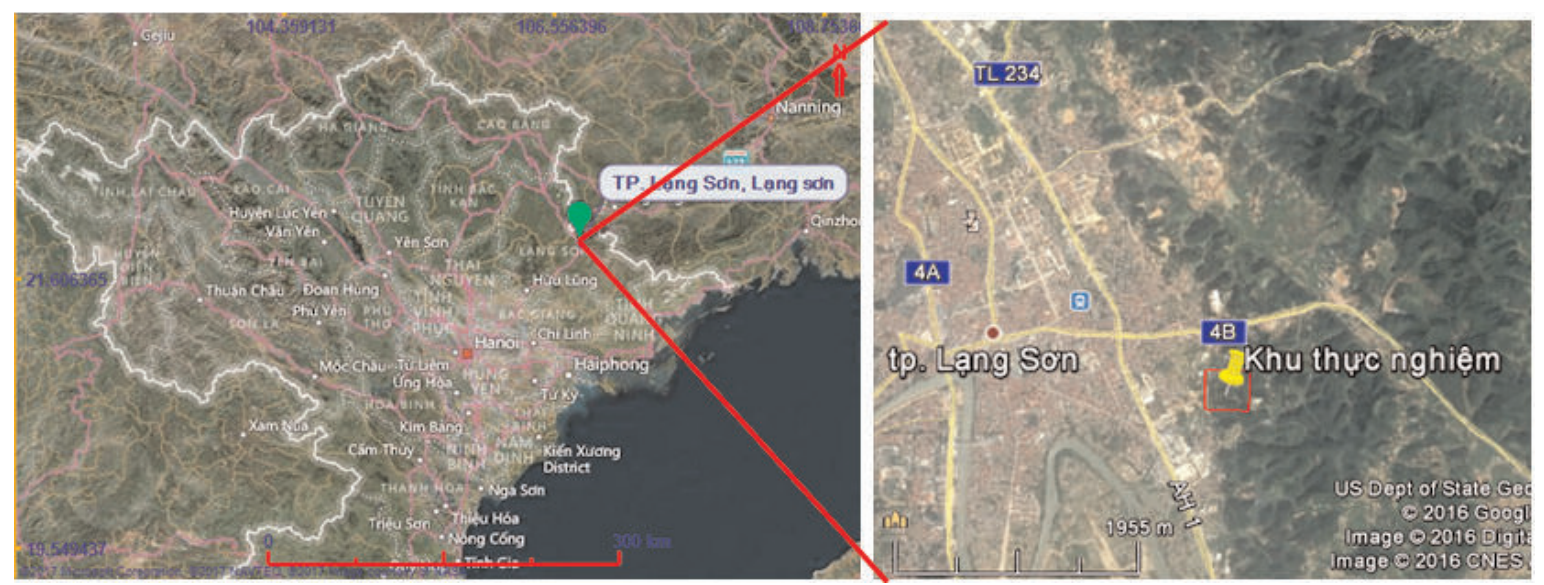

Hình 4: Bản đồ vị trí khu vực thử nghiệm 


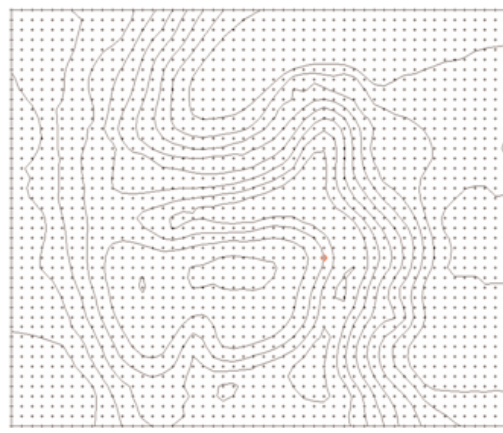

Hình 5: Mô hình DEM chuẩn

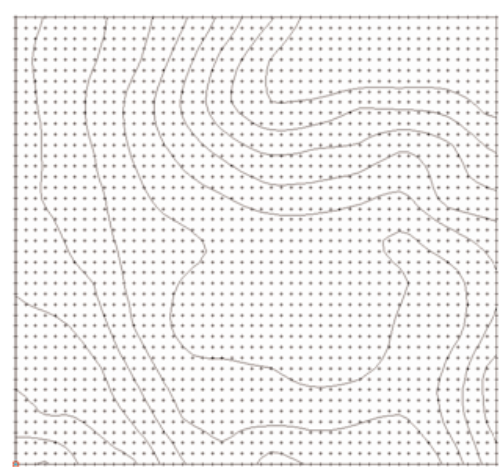

Hình 7: Mô hình DEM thử nghiệm

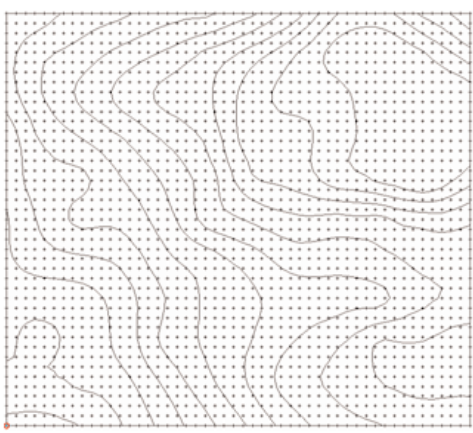

Hình 6: Mô hình ASTER GDEM (DEM gốc)

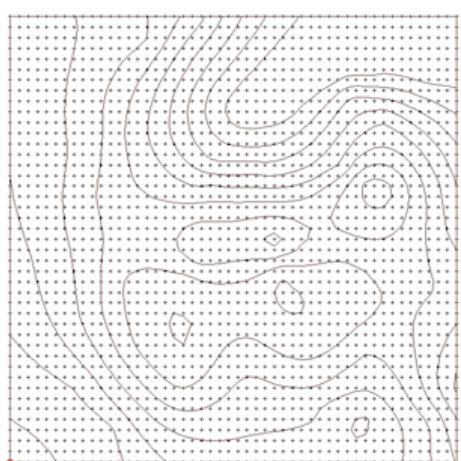

Hình 8: Mô hình DEM sau xử lý

Bảng 1: Kết quả đánh giá độ chính xác của các DEM

\begin{tabular}{|c|c|c|c|c|c|c|c|}
\hline STT & $\mathrm{DEM0}(\mathrm{m})$ & DEM1(m) & $\Delta \mathrm{h} 1(\mathrm{~m})$ & $\mathrm{DEM} 2(\mathrm{~m})$ & $\Delta \mathrm{h} 2(\mathrm{~m})$ & DEM3(m) & $\Delta \mathrm{h} 3(\mathrm{~m})$ \\
\hline 1 & 278.58 & 270.985 & -7.595 & 274.188 & -4.392 & 274.055 & -4.525 \\
\hline 2 & 278.736 & 270.954 & -7.782 & 274.423 & -4.313 & 273.943 & -4.793 \\
\hline 3 & 278.773 & 270.919 & -7.854 & 274.671 & -4.102 & 273.897 & -4.876 \\
\hline 4 & 278.8 & 270.945 & -7.855 & 274.937 & -3.863 & 274.109 & -4.691 \\
\hline 5 & 277.898 & 271.102 & -6.796 & 275.224 & -2.674 & 274.758 & -3.14 \\
\hline 6 & 277.931 & 271.366 & -6.565 & 275.538 & -2.393 & 275.731 & -2.2 \\
\hline 7 & 277.43 & 271.693 & -5.737 & 275.883 & -1.547 & 276.827 & -0.603 \\
\hline 8 & 277.904 & 272.092 & -5.812 & 276.265 & -1.639 & 277.914 & 0.01 \\
\hline 9 & 279.138 & 272.612 & -6.526 & 276.691 & -2.447 & 278.876 & -0.262 \\
\hline 10 & 281.318 & 273.278 & -8.04 & 277.181 & -4.137 & 279.686 & -1.632 \\
\hline 11 & 284.06 & 274.062 & -9.998 & 277.831 & -6.229 & 280.46 & -3.6 \\
\hline 12 & 286.492 & 274.969 & -11.523 & 278.912 & -7.58 & 281.347 & -5.145 \\
\hline 13 & 288.392 & 276.055 & -12.337 & 280.451 & -7.941 & 282.537 & -5.855 \\
\hline 14 & 292.124 & 277.37 & -14.754 & 282.11 & -10.014 & 284.185 & -7.939 \\
\hline$\ldots$ & $\ldots$ & $\ldots$ & $\ldots$ & $\ldots$ & $\ldots$ & $\ldots$ & $\ldots$ \\
\hline 10000 & 314.79 & 312.588 & -2.202 & 313.533 & -1.257 & 313.103 & -1.687 \\
\hline $\mathrm{mDEM}$ & & 8.642 & & 6.121 & & 5.701 & \\
\hline
\end{tabular}

độ chính xác mô hình vào khoảng $5.7 \mathrm{~m}$. và $7 \%$. Phản ánh trực quan từ kết quả phân Với giá trị này có thể tính toán được mức cải tích trên đây có thể được nhìn thấy từ các thiện so với $\mathrm{DEM} 1$ và $\mathrm{DEM} 2$ lần lượt là $34 \%$ hình $5,6,7$ và 8 . Chúng ta thấy rằng hình 
dạng địa hình thông qua đường đồng mức của DEM sau xử lý có độ tương tự cao hơn so với $D E M$ gốc và $D E M$ thử nghiệm.

\section{Kết luận}

Bài báo này đề xuất một phương pháp nhằm nâng cao độ chính xác của $\mathrm{DEM}$ có độ chính xác thấp dựa trên các điểm đo bổ sung có độ chính xác cao hơn thông qua phép lọc Kalman và phép làm trơn RTS. Việc thử nghiệm được thực hiện trên mô hình DEM toàn cầu ASTER GDEM kết hợp với các điểm đo thực địa tại khu vực Lạng Sơn. Kết quả tính toán thử nghiệm chỉ ra rằng với phương pháp đã đề xuất, mô hình số độ cao gốc được cải thiện đáng kể về độ chính xác sau khi xử lý.

Trong các nghiên cứu tới, việc phát hiện và loại bỏ sai số thô trong các trị đo sẽ được tập trung nghiên cứu. Phạm vi khu thực nghiệm sẽ được mở rộng để có những đánh giá tin cậy hơn về phương pháp đã đề xuất. $O$

\section{Tài liệu tham khảo}

[1]. P.L. Guth, Geomorphometric comparison of Aster GDEM and RSTM, ASPRS/CaGIS 2010 Fall Specialty
Conference, November 15-19, 2010 Orlando, Florida

[2]. F. Bignone a, H. Umakawa, Assessment of ALOS PRISM Digital Elevation Model extraction over Japan, The International Archives of the Photogrammetry, Remote Sensing and Spatial Information Sciences. Vol. XXXVII. Part B1. Beijing 2008

[3]. Nicholas J. Tate, Smoothing/filtering LiDAR digital surface models. Experiments with loess regression and discrete wavelet, Journal of Geographical Systems, 7: 273-290 (2005)

[4]. Ping Wang, Applying two dimensional Kalman filter for digital terrain modeling, ISPRS, 32(4), Stuttgart (1998)

[5]. Kalman, R.E. A new research approach to Linear Filtering and Prediction Problem. Journal of Basic Enginerring, 1960, Vol.82, Series D, 35-45.

[6]. Rauch, H.; Tung, F. and Striebel, C. Maximum likelihood estimates of linear dynamic systems, AIAA J., 1965, Vol.3, No.8, 1445-1450. 0

\section{Summary}

\section{Improving the accuracy of Digital Elevation Model by using Kalman filter and Rauch-Tung-Striebel smoother}

Duong Thanh Trung, Dao Van Khanh, Truong Minh Hung, Hanoi University of Mining and Geology

\section{Nguyen Duc Manh, Vietnam Institute of Geodesy and Cartography}

Digital Elevation Model (DEM) is necessary for many applications such as earth science, natural resources and environment. Generally, there are two methods to build DEM including conventional land surveying and remote sensing technique. The fact indicates that the conventional land surveying method produce more accurate DEM $(2 \mathrm{~cm}-1 \mathrm{~m})$ but it is high cost and time consuming. Conversely, the remote sensing method, particularly, using satellite images can produce DEM with low cost but low accuracy $(5-30 \mathrm{~m})$. This article propose a method to improve the efficiency of producing DEM by integration of DEM generated by remote sensing technology and DEM generated by land surveying via Kalman filter and Rauch-Tung-Striebel smoother. $\bigcirc$ 